\title{
Timekeeping with electron spin states in diamond
}

\author{
J. S. Hodges, ${ }^{1, *}$ N. Y. Yao, ${ }^{2}$ D. Maclaurin, ${ }^{2}$ C. Rastogi,${ }^{3}$ M. D. Lukin, ${ }^{2}$ and D. Englund ${ }^{1,3, \dagger}$ \\ ${ }^{1}$ Department of Electrical Engineering, Columbia University, New York, New York 10027, USA \\ ${ }^{2}$ Department of Physics, Harvard University, Cambridge, Massachusetts 02138, USA \\ ${ }^{3}$ Department of Applied Physics and Applied Mathematics, Columbia University, New York, New York 10027, USA
}

(Received 4 September 2011; revised manuscript received 27 November 2012; published 28 March 2013)

\begin{abstract}
Frequency standards based on atomic states, such as $\mathrm{Rb}$ or Cs vapors, or single-trapped ions, are the most precise measures of time. Here we propose and analyze a precision oscillator approach based upon spins in a solid-state system, in particular, the nitrogen-vacancy defect in single-crystal diamond. We show that this system can have stability approaching portable atomic standards and is readily incorporable as a chip-scale device. Using a pulsed spin-echo technique, we anticipate an Allan deviation of $\sigma_{y}=10^{-7} \tau^{-1 / 2}$ limited by thermally-induced strain variations; in the absence of such thermal fluctuations, the system is limited by spin dephasing and harbors an Allan deviation nearing $\sim 10^{-12} \tau^{-1 / 2}$. Potential improvements based upon advanced diamond material processing, temperature stabilization, and nanophotonic engineering are discussed.
\end{abstract}

DOI: 10.1103/PhysRevA.87.032118

PACS number(s): 06.20.fb, 76.30.-v, 71.55.Cn

\section{INTRODUCTION}

Atomic clocks are the most accurate systems for measuring time and frequency. They are used in a broad array of applications, ranging from communication and computation (e.g., precise broadcast frequency standards) to navigation [e.g., the global positioning system (GPS)]. Modern frequency standards derive their stability from the precisely measured internal hyperfine level splittings of atoms of cesium, rubidium, or hydrogen. When an oscillating magnetic field is resonant with the energy difference of these internal states, a change in population between levels alters the radio frequency or optical absorption. Standard lock-in techniques modulate the driving frequency and monitor the absorption as a correction for a tunable active reference oscillator, e.g., a quartz crystal, thus stabilizing it to the atomic resonance line [1]. Recent experiments on single-trapped ions [2] and on ensembles of atoms trapped in optical lattices $[3,4]$ have far exceeded the international cesium standard, enabling the observation of general relativity corrections at distances of a few meters [5]. Such precision, however, comes at the expense of mobility, as the infrastructure for these standards encompasses several tens of cubic meters of space. At the other extreme, portable standards based on rubidium vapor cells provide excellent stability for time scales ranging from 1 to $10^{4} \mathrm{~s}$ and find usage in satellites, laboratory equipment, and cellular communications [6]. Mobile devices, which typically do not contain their own precision standards, can share GPS time signals for maintaining communication standards, but when the external lock signal is obstructed, a precise local frequency standard with minimal drift is necessary to maintain synchronization.

To address this need, several groups have miniaturized these atomic standards on chip through the aid of modern microfabrication techniques applied to detectors and lasers

\footnotetext{
*Present address: The MITRE Corporation, 200 Forrestal Road, Princeton, NJ 08540, USA; jsh58@ columbia.edu

${ }^{\dagger}$ Present address: Research Laboratory of Electronics, Massachusetts Institute of Technology, Cambridge, MA 02139, USA; englund@mit.edu
}

[7-9]. Here, we propose a solid-state alternative based upon electronic spin states in the negatively charged nitrogenvacancy center (NV) center in diamond. This diamond system offers a host of potential advantages, while at the same time pointing to some unique challenges related to the complex solid-state environment. First, single-crystal diamond can be grown into a micron-scale, radiation hard chip, which makes it portable and well suited for integration in a semiconductor fabrication process [10]. Second, this solid-state system derives its performance as a clock from exceptional spin lifetimes of the NV [11] and closely resembles atomic and molecular systems. The optical detection of the NV center also increases the signal-to-noise ratio of solid-state standards based on inductive detection [12]. Compared to vapor cells, this standard does not suffer from Doppler or collisional broadening. However, because of the complex mesoscopic environment, both single and ensembles of NV centers exhibit increased homogeneous linewidths compared to atomic standards [13]. The higher density of defects in solids allows for a comparable frequency stability in smaller sensor volumes when ensemble effects can be mitigated. Similar to atomic clocks, temperature fluctuations can degrade the clock stability. For the NV center, these temperature changes induce a shift in the resonance frequency of the entire ensemble.

In this paper, we analyze both the potential advantages and limitations of an NV-based frequency standard and precision oscillator and describe techniques to compensate for thermally-induced strain variations which limit the clock's performance. Using such temperature stabilization methods, we estimate a stability, expressed as an Allan deviation, which approaches $\sigma_{y} \approx 2 \times 10^{-10}$ at $1 \mathrm{~s}$ of averaging for a 0.1 $\mathrm{mm}^{3}$ diamond sample with an ensemble of NVs. Paired with potentially modest operational power requirements, this NV clock could result in a new generation of portable solid-state precision oscillator.

Our paper is organized as follows. In Sec. II, we begin with a brief review of the NV-level structure and fluorescence properties, before moving on to a discussion of the zero-field splitting as a possible frequency standard. We then propose a pulsed approach toward an NV-based clock, offering a 
comparison of sensitivities along the way. In Sec. III, we discuss the effects of dephasing and inhomogeneous broadening, which will limit the achievable clock sensitivity. In Sec. IV, we address the dominant source of instability, namely, the fact that the zero-field splitting of the NV exhibits a strong temperature dependence. We discuss the origin of this temperature dependence and show that a consistent picture arises if we assume that it results from temperature-induced lattice modulations. We then propose two possible solutions to this challenge, first, by synchronizing two NV clocks and second, by canceling the temperature dependence using engineered strain. Finally, in Sec. V, we conclude by comparing the NV frequency standard with other state-of-the-art chip-based clocks.

\section{THE NV ZERO-FIELD SPLITTING AS A FREQUENCY STANDARD}

An atomic clock derives its stability from the large quality factor $Q=v / \Delta v$ of the probed resonance, with narrow linewidth $\Delta v$ being much smaller than the resonant frequency $v$. In the solid state, we desire (i) a microwave transition $(v \sim \mathrm{GHz})$ that also exhibits a large $Q$; (ii) a resonance that does not vary due to material or fabrication processes; and (iii) a precise method of measuring population changes. The NV center in diamond satisfies these criteria by having a ground state spin triplet characterized by long ( $>1 \mathrm{~ms})$ coherence times, a ground state crystal field splitting with an intrinsic resonance frequency near $2.870 \mathrm{GHz}$, which is independent, to lowest order, of applied magnetic field, and spin states that are optically polarizable and detectable on single defect length scales $(\sim 1 \mu \mathrm{m})$.

We construct a simple model for the relevant NV spin dynamics by considering a Hamiltonian for the lowest and first excited triplet state as well as two metastable singlet states. Figure 1(a) shows the relevant spin sublevels: 0, 1, 2,3 , and $S$, which are two of the ground state spin sublevels, two of the excited state sublevels, and a metastable singlet state, respectively. For the purposes of a frequency standard, we monitor the fluorescence response of only the ground state triplet sublevels (0 and 1) to resonant microwave excitation, yielding the ground state Hamiltonian [14]

$$
\begin{aligned}
\mathbf{H}_{\mathrm{gs}}= & \left(D_{\mathrm{gs}}+d_{\|} \sigma_{z}\right) S_{z}^{2}+g \mu_{b} \vec{S} \cdot \vec{B} \\
& +d_{\perp} \sigma_{x}\left(S_{x} S_{y}+S_{y} S_{x}\right)+d_{\perp} \sigma_{y}\left(S_{x}^{2}-S_{y}^{2}\right) .
\end{aligned}
$$

Here, $d_{\|, \perp}$ are the ground state electric dipole moment components along and perpendicular to the $C_{3 v}$ symmetry axis of the defect. $D_{\mathrm{gs}}$ is the ground state crystal field splitting $(2.870 \mathrm{GHz}), \mu_{b}$ is the Bohr magneton, and $g$ is the Landé factor [15]. $S_{k}$ are spin-1 operators in the $k=\{x, y, z\}$ directions. The local electric field vector, induced by crystal strain, is $\vec{\sigma}$. We assume there is no applied external electric field. In the limit of static applied magnetic and instrinsic electric fields that change the magnetic sublevel energies much smaller than $D_{\mathrm{gs}}$, the eigenfunctions are those of the $S_{z}$ operator, as shown in Fig. 1.

A driving field at frequency $\omega$ induces electron spin resonance (ESR) transitions between $|0\rangle$ and $| \pm 1\rangle$. On resonance ( $\omega \approx D_{\mathrm{gs}}$ ), the photoluminescence (PL) decreases and provides a feedback signal with which to lock $\omega$ to $D_{\mathrm{gs}}$. The dynamics are best viewed as a response to a time-varying magnetic field $B_{1}=2 b_{1} \cos (2 \pi \omega t) \hat{x}$; transforming $H_{\mathrm{gs}}$ into the interaction frame defined by the operator $V=e^{2 \pi i \omega t S_{z}^{2}}$ and performing the rotating wave approximation yields

$$
\mathbf{H}_{\mathrm{gs}}^{\prime}=\left(D_{\mathrm{gs}}+d_{\|} \sigma_{z}-\omega\right) S_{z}^{2}+g \mu_{b} B_{z} S_{z}+g \mu_{b} b_{1} S_{x} .
$$

The relaxation rates of the excited triplet and singlet states, shown in Fig. 1(a), play an important role in the optical pumping and spin measurement [16]. We model the total magneto-optical response using a master equation approach:

$$
\dot{\rho}=\frac{1}{i \hbar}\left[H_{\mathrm{gs}}^{\prime}, \rho\right]+\sum_{k} L_{k} \rho L_{k}^{\dagger}-\frac{1}{2} L_{k}^{\dagger} L_{k} \rho-\frac{1}{2} \rho L_{k}^{\dagger} L_{k},
$$

(b)
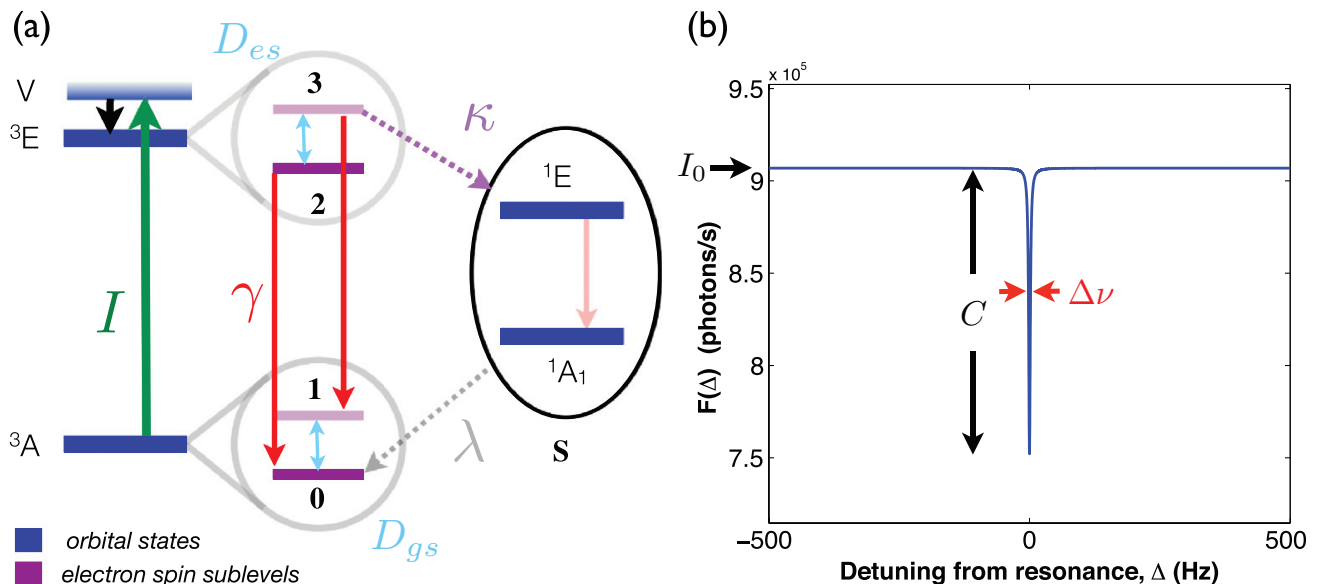

FIG. 1. (Color online) Nitrogen-vacancy-center energy levels and resonant response. (a) The lowest lying triplet $\left({ }^{3} E,{ }^{3} A\right)$ and singlet $\left({ }^{1} E\right.$, ${ }^{1} A$ ) orbital states of the $\mathrm{NV}^{-}$center. $I, \gamma, \kappa$, and $\lambda$ represent the absorption, PL, intersystem crossing, and deshelving rates, respectively. Sublevels 0 and 2 are $S_{z}$ eigenstates $|0\rangle$, whereas 1 and 3 are $| \pm 1\rangle$, with the degeneracy lifted by small crystal strain or applied magnetic field. The model is simplified by approximating both singlet states as a single metastable level. (b) The steady-state fluorescence emission of the NV center under continuous optical and microwave irradiation, detuned from resonance by $\Delta$. The PL response function, as derived from a master equation approach, is approximated by a Lorentzian: $F(\Delta)=I_{0}\left(1-\frac{C \delta v^{2}}{(\Delta / \pi)^{2}+\delta v^{2}}\right)$, where $C$ is the modulation depth and $\delta v$ is the FWHM [18]. 
where $\rho$ is the density operator for the NV-center ground, excited triplet, and effective singlet states. The jump operators $L_{k}$ have magnitudes corresponding to relaxation rates $\sqrt{r_{k}}$ (see Appendix A). The solution to the equation yields the total magneto-optical response for both continuous and pulsed excitation, allowing for a numerical assessment of the clock sensitivity with respect to changes in the electromagnetic fields.

\section{A. NV-based clock with continuous excitation and readout}

Under continuous excitation of optical and microwave fields, the NV frequency standard closely resembles a twoisotope Rb standard. Spin-dependent PL occurs under nonresonant absorption of green light of intensity $I$. Application of a microwave field of intensity $\Omega=g \mu_{b} b_{1}$, detuned from resonance by an amount $\Delta=D_{\mathrm{gs}}-\omega$, causes a broad, phonon-assisted PL: $F(I, \Omega, \Delta)=\gamma \rho_{22}^{\text {ss }}+\frac{\gamma^{2}}{\kappa+\gamma} \rho_{33}^{\text {ss }}$. Here $\rho_{22}^{\text {ss }}$ and $\rho_{33}^{\text {ss }}$ represent the populations of the first excited state spin sublevels in the steady state, as analytically derived from a master equation (see Appendix A). Figure 1(b) shows the typical response of $F$ for varied detunings, which displays a Lorentzian line shape. The stability of the clock can be derived from the resonance curve by considering the Allan variance

$$
\sigma_{y}(\tau)=\frac{1}{2 \pi Q} \frac{1}{(S / N)} \frac{1}{\sqrt{\tau}},
$$

where $\tau$ is the averaging time and $S / N$ is the signal-to-noise ratio, which depends on both the photon shot noise as well as the imperfect modulation of the resonance (i.e., $C \neq 1$ ). The intrinsic linewidth is limited by the paramagnetic and nuclear spin environments which fluctuate during the measurement. This linewidth broadens if the microwave and optical transitions are driven near saturation ("power broadening"); however, higher pump powers also increase the depth of the $\operatorname{dip}(C \rightarrow 1)$. Indeed, far below optical saturation, the PL rate is sufficiently small, and the modulation depth $(C)$ is reduced, so as to cause a decrease in the stability per averaging time. As $Q$ is the most important factor for stability, there exists an optimal condition, which balances the line broadening with the reduced signal. Under these conditions, we estimate a linewidth of $3.6 \mathrm{MHz}\left(T_{2}^{*}=88 \mathrm{~ns}\right)$, an off-resonance fluorescence rate of $\sim 9400$ photon/s (accounting for a finite detector efficiency), and a $17 \%$ modulation depth. With these parameters, $\sigma_{y}(\tau)=8.124 \times 10^{-5} \tau^{-1 / 2}$ for a single NV.

In short, single or ensembles of NV centers lack sufficient stability when monitoring the PL response continuously. This results from the fact that the laser excitation must be reduced far below saturation so that optical power broadening reaches the homogeneous linewidth. At such low pump powers, the fluorescent photon flux is so small that the gains in $Q$ are offset by losses in $S / N$. As seen in magnetometry studies of the NV [17], a pulsed microwave excitation scheme, which monitors transient fluorescence behavior, can drastically improve the performance beyond the naive continuous excitation or detection method.

\section{B. NV-based clock with pulsed excitation and readout}

We now consider monitoring the NV crystal field splitting $\left(D_{\mathrm{gs}}\right)$ in a pulsed fashion, akin to the Ramsey spectroscopy used in atomic clocks. The standard two-pulse Ramsey sequence, with time separation $T$, imprints a phase proportional to the frequency drift $\delta \omega$ between two hyperfine states of the atomic system. The PL response varies sinusoidally with $\delta \omega T$ and can be linearized to provide a passive standard with frequency uncertainty set by $T^{-1}$. However, if the system does not remain coherent for times $T \gg T_{c}$ (coherence time), the modulation of the PL response does not persist. In atomic vapor cells, trapped ions, or atomic fountains, $T_{c}$ can be greater than a second due to a complex infrastructure involving magnetic shielding and the minimization of atomic collisions. In the NV system, a spin-bath environment limits $T_{c}$ to tens of microseconds in a typical sample. Quantum memories and ac magnetometers often employ a Hahn echo sequence to extend this coherence time to $T_{2}$ by removing slowly varying magnetic fields; however, for frequency standards based on $m_{f}=0$ "clock" states, the additional $\pi$ pulse of the Hahn echo would completely remove the phase accumulation associated with frequency drift, making it useless for timekeeping. Luckily, the $S=1$ nature of the NV allows for a modified echo sequence, which yields a PL signal proportional to the drift for $T \sim T_{2}$.

In particular, we begin with the usual spin-echo sequence unitary propagator,

$$
\begin{aligned}
U_{\text {echo }}= & e^{-i(\pi / 2) S_{x}} e^{-i\left(\delta \omega S_{z}^{2}+b S_{z}\right) \tau} e^{i(\pi / 2) S_{x}} e^{i(\pi / 2) S_{x}} e^{-i(\pi / 2) S_{x}} \\
& \times e^{-i\left(\delta \omega S_{z}^{2}+b S_{z}\right) \tau} e^{-i(\pi / 2) S_{x}}=W W^{\prime},
\end{aligned}
$$

where

$$
\begin{aligned}
& W=e^{-i\left(\delta \omega \hat{X}-b S_{y}\right) \tau}, \quad W^{\prime}=e^{-i\left(\delta \omega \hat{X}+b S_{y}\right) \tau}, \text { and } \\
& \hat{X}=\left[\begin{array}{ccc}
\frac{1}{2} & 0 & -\frac{1}{2} \\
0 & 1 & 0 \\
-\frac{1}{2} & 0 & \frac{1}{2}
\end{array}\right] .
\end{aligned}
$$

Since $\left[\hat{X}, S_{y}\right]=0$, we can write the product of $W$ and $W^{\prime}$ as the sum of exponential arguments such that $U_{\text {echo }}=e^{-2 i \delta \Omega \tau \hat{X}}$. To take advantage of this propagator we must first apply a pulse that creates a superposition of $\hat{X}$ eigenstates with differing eigenvalues; starting from the optically pumped $|0\rangle$ state, we accomplish this by applying a $\frac{\pi}{4}$ pulse about the $\hat{x}$ axis: $U_{45}=$ $e^{-i(\pi / 4) S_{x}}$, such that $U_{45}|0\rangle=\frac{1}{\sqrt{2}}(|0\rangle-i|+\rangle)$, where $|+\rangle$ is the symmetric superposition of the \pm 1 eigenstates of $S_{z}$. This yields the state prior to clock measurement: $U_{\text {echo }} U_{45}|0\rangle=$ $\frac{1}{\sqrt{2}}\left(-i e^{i \delta \Omega \tau}|+\rangle+e^{-i \delta \Omega \tau}|0\rangle\right)$. Thus, the overall clock sequence for our spin- $1 \mathrm{NV}^{-}$center is

$$
\left.\left.\left.U_{\text {clock }} \equiv \frac{\pi}{4}\right)_{x} \stackrel{\tau}{\longrightarrow} \pi\right)_{-x} \stackrel{\tau}{\longrightarrow} \frac{\pi}{4}\right)_{x},
$$

where the notation $\vartheta)_{\phi}$ indicates a pulse with a flip angle $\vartheta$ and phase $\phi$.

As seen in Eq. (2), the term proportional to $S_{z}^{2}$ is sensitive to the frequency drift $(\delta v)$, whereas the term proportional to $S_{z}$ varies with $\vec{B}$. In addition, hyperfine interactions with nearby nuclear spins have a secular correction term to the Hamiltonian $\propto A_{\|} S_{z} I_{z}$. By modifying the flip angle of the first and last pulses of the traditional spin- echo sequence from $\pi / 2$ to $\pi / 4$, 

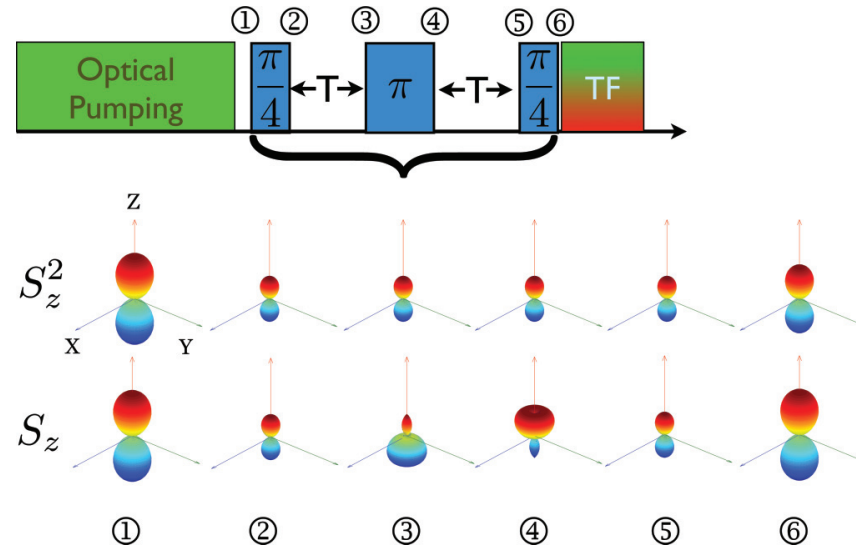

FIG. 2. (Color online) The spin-1 ground state $|0\rangle$ state is prepared by optical pumping. $45^{\circ}$ rotations ensure that the $S_{z}$ term evolution averages within the echo sequence, while the $S_{z}^{2}$ does not, as illustrated in the Euler projections of the state (inset). These projections are to show the equipotential surface plots, $\mathcal{E}(\kappa, \varphi)$ the density matrix element $|0\rangle\langle 0|$ for each point of the sequence after undergoing rotations $\kappa$ and $\varphi$ about the $S_{y}$ and $S_{z}$ axes, respectively: $\mathcal{E}=\left\langle 0\left|R^{\dagger} \rho R\right| 0\right\rangle, R=e^{i \varphi S_{y}} e^{i \kappa S_{z}}$ and provide a visual representation of the coherences between states, akin to the Bloch sphere for two-level systems [19]. Note that the $S_{z}$ term is completely refocused by the echo sequence, while the $S_{z}^{2}$ operator evolves, resulting in a different magnitude between the first and sixth states. A transient fluorescence (TF) measurement records the photocurrent for $\sim 300 \mathrm{~ns}$ timed with a pulse of green light.

as shown in Fig. 2, the phase accumulation due to the $S_{z}$ term cancels, while that from the $S_{z}^{2}$ terms add over the duration $2 T$. For simplicity, we monitor the eigenkets of $S_{z}$ to describe the clock, assuming perfect microwave pulses and no optical illumination. First, optical pumping of the NV prepares the initial state $\left|\psi_{0}\right\rangle=\left|m_{s}=0\right\rangle$. Evolution under our drift clock sequence $U_{\text {clock }}$ gives

$\left|\psi_{f}\right\rangle=U_{\text {clock }}\left|\psi_{0}\right\rangle=\frac{1}{\sqrt{2}} \sin (\phi)|0\rangle-\frac{1}{2} \cos (\phi)(|+1\rangle+|-1\rangle)$,

where $\phi=\left(D_{\mathrm{gs}}-\omega\right) T=\delta \omega T$.

Unlike the steady-state case, the NV spin is prepared in a state used for timekeeping, then measured optically, without simultaneous microwave excitation. The transient PL response of the NV center can be modeled using projective measurements. The operator $\hat{M}$ describes the spin expectation value for a PL measurement [20], $\hat{M}=a|0\rangle\langle 0|+b(|+1\rangle\langle+1|+$ $|-1\rangle\langle-1|)$, where $a$ and $b$ are independent Poisson random variables. The fractional frequency deviation varies as the quantum observable, $\hat{M}$ for our state $\left|\psi_{f}\right\rangle$ according to

$$
\frac{\delta \omega}{\omega_{0}}=\frac{1}{\omega_{0}} \frac{\Delta \hat{M}}{|\partial\langle\hat{M}\rangle / \partial \omega|},
$$

where $\Delta \hat{M}^{2}=\left\langle\hat{M}^{2}\right\rangle-\langle\hat{M}\rangle^{2}$ is the variance of the operator. For room temperature spin readout of the NV, $2 a \approx 3 b$ [21]. By calculating the moments of $\hat{M}$ and by assuming we accumulate $M^{\prime}$ independent measurements (e.g., total sampling time $\tau=$
$\left.M^{\prime} T\right)$, we arrive at

$$
\left\langle\frac{\delta \omega}{\omega_{0}}\right\rangle_{M^{\prime}}=\frac{\xi}{D_{\mathrm{gs}} \sqrt{T \tau}}
$$

with $\xi \approx 5$ due to a combination of imperfect spin readout (i.e., $b \neq 0$ ), imperfect collection efficiency $(a \approx 0.031)$, and a small ratio, $\lambda / \gamma$, of the metastable deshelving rate to the radiative lifetime (see Appendix B). Taking $T=T_{2} \approx 1 \mathrm{~ms}$ and $D_{\mathrm{gs}}=2870 \mathrm{MHz}$ gives a deviation of $\delta \omega / \omega_{0}=8.8 \times$ $10^{-9} / \sqrt{\tau}$ for a single NV. Thus, the pulsed NV frequency standard dramatically outperforms the continuous wave (CW) scheme. A comparison of the two schemes for different defect concentrations, along with established standards, is shown in Fig. 3. Note that all optical collection efficiencies (assumed to be $\sim 0.2 \%$ of the total radiated photons) do not yet include cavity or resonant enhancements; this is, in fact, an area of current research [22] and will likely lead to further improvements. Moreover, dynamical decoupling techniques, developed for nuclear magnetic resonance spectroscopy and, more recently, quantum information processing, have already shown promise in extending phase coherence times of NV centers [23-25].

The stability for a single NV center can be scaled by collecting the fluorescence from an ensemble of $N$ noninteracting NV centers, yielding a $1 / \sqrt{N}$ enhancement. To make a reasonable estimate of $N$, we start with the density of pure diamond: $1.74 \times 10^{23} \mathrm{C}$ atoms $/ \mathrm{cm}^{3}$. At an $\mathrm{NV}^{-}$defect fraction of $10^{-11}$ [0.01 parts per $\left.10^{9}(\mathrm{ppb})\right]$, the density of defects is $1.74 / \mu \mathrm{m}^{3}$, consistent with commercially available samples. For a $1 \mathrm{~mm}^{3}$ sample, the proposed $1 / \sqrt{N}$ enhancement would yield a

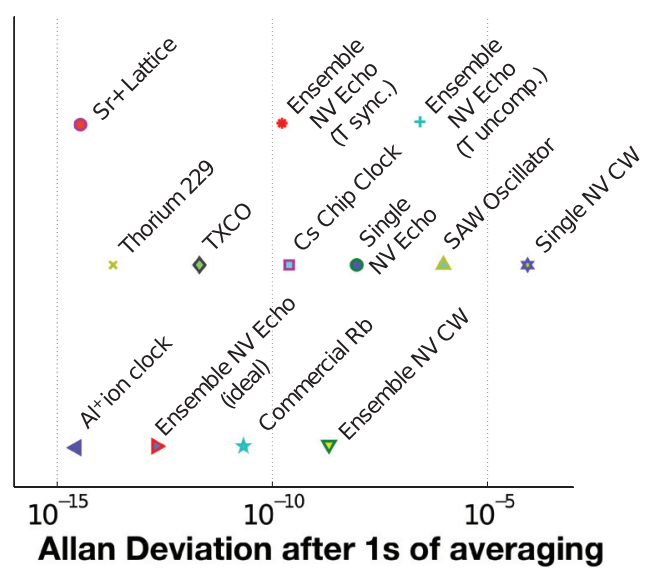

FIG. 3. (Color online) Allan deviation for atomic and solidstate standards. Note that in moving from a $\mathrm{CW}$ scheme with a single NV center to an ensemble of centers with pulsed excitation and detection, we gain almost six decades of improvement. Cited deviations are as follows: Al-ion clock [2,5]; Sr lattice clock [4]; thorium clock (theoretical) [35]; Cs chip clock [7]; TXCO and commercial Rb figures available from Stanford Research Systems (www.thinksrs.com); surface acoustic wave (SAW) oscillator is quote from Epson Toyocom Corporation EG-4101/4121CA datasheet. Pulsed echo NV samples are assumed to have $0.01 \mathrm{ppb} N \mathrm{~N}$ center concentrations for a $1 \mathrm{~mm}^{3}$-sized sample. Note the difference of roughly three orders of magnitude achieved by synchronizing the temperature compared to the uncompensated thermal drift. The ideal echo scheme assumes no temperature dependence, with sensitivity limited by the spin lifetime. 


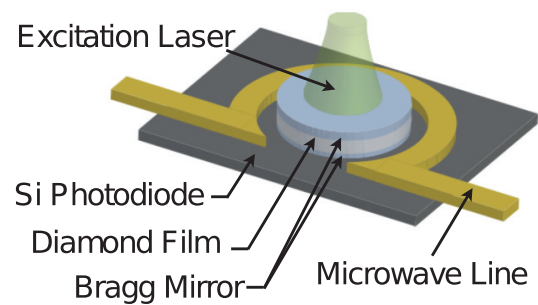

FIG. 4. (Color online) Schematic of the diamond frequency standard. A thin $(100 \mu \mathrm{m})$ diamond chip is surrounded by dielectric stacks (Bragg reflectors) on both sides to create a resonant cavity for $532 \mathrm{~nm}$ excitation in order to reduce the power requirements. On-chip $532 \mathrm{~nm}$ excitation comes from a doubled $1064 \mathrm{~nm}$ surface emitting laser (not shown). Silicon photodetectors underneath the diamond serves to collect emission. It may also be advantageous to collect emission from the near side of the device [22]. Microwaves, which address the NV magnetic sublevels, are applied to the entire sample by a planar stripline.

fractional frequency deviation of $2 \times 10^{-13} / \sqrt{\tau}$ for the pulsed scheme and $2 \times 10^{-9} / \sqrt{\tau}$ for the $\mathrm{CW}$ scheme; thus, a diamond film of thickness $\sim 100 \mu \mathrm{m}$ with a surface area $\sim 1 \mathrm{~mm}^{2}$, as depicted in Fig. 4, gives $\sigma_{y}^{\text {pulsed }} \sim 6.7 \times 10^{-13} / \sqrt{\tau}$. Further increasing the defect density to $1 \mathrm{ppb}$ would improve all values by another order of magnitude, but dephasing effects may start to arise owing to interactions with other paramagnetic impurities [26]. Next, we will consider limitations of the NV frequency standard imposed by dephasing, ensemble effects, and temperature.

\section{DEPHASING AND INHOMOGENEOUS BROADENING}

Dephasing. The coherent evolution of the NV center is strongly affected by interactions with its local environment, meaning that the $T^{-1 / 2}$ scaling of Eq. (15) cannot be maintained for all times. Unlike an atomic vapor, where collisions and stray magnetic fields dominate the dephasing, in the case of the NV, impurities in the solid-state lattice limit the coherence. However, excellent material growth [11] and precision implantation [10,27] can yield crystals which are largely free of paramagnetic impurities. For a single NV center in an ultrapure diamond sample, spin echo (of duration 2T) can further improve the observable coherence with a scaling $e^{-\left(2 T / T_{2}\right)^{n}}$, where the exponent $n \approx 3$ is related to the nuclear spin bath $[13,28]$. In an ensemble of NVs, where each center has a different bath, and hence, a different $T_{2}$ time, the net effect results in an "averaged" time constant, $T_{e}$, and an exponent between $n=1$ and $n=2$, depending on the NV density [29]. We also note that owing to imperfect conversion efficiency, the NV density in the sample is a fraction of the total number of substitutional nitrogen impurities. Thus, a heavily doped sample, while improving the signal-to-noise ratio, can reduce the overall ensemble coherence time $T_{e}$. Based on the estimates in Ref. [26], with an NV density of $10^{17} / \mathrm{cm}^{3}$, a marginal modification can be expected; Fig. 3 shows the Allan deviation floor due to such dephasing considerations.

Inhomogeneous broadening. By taking advantage of spin echo, the pulsed NV frequency standard shows a favorable Allan deviation when compared to similarly sized atomic standards. As discussed above, considering an ensemble of defects provides a straightforward route to improving the Allan variance; however, the local strain field within the crystal may vary from center to center resulting in inhomogeneous broadening. Recent room-temperature experiments on bulk diamond (defect fraction of $\sim 10^{-13}$ ) have observed $50-100 \mathrm{kHz}$ variations in the NV $D_{\mathrm{gs}}$ of centers spaced at $\sim 1 \mu \mathrm{m}$ [30]. These parameters would imply that for an NV-ensemble clock, the sensitivity is, in fact, limited by the inhomogeneously broadened ensemble linewidth $T_{2 \text {,ens }} \sim 10-20 \mu \mathrm{s}$. This would reduce our estimated stability by nearly an order of magnitude and motivates the development of modern chemical vapor deposition growth and annealing techniques, which may be able to alleviate such local strain fluctuations [31].

\section{TEMPERATURE FLUCTUATIONS}

One of the more challenging aspects of an NV frequency standard is the fact that the resonance frequency corresponding to $D_{\mathrm{gs}}$ varies as function of temperature, $\theta$. At room temperature, recent ensemble studies have observed $d D_{\mathrm{gs}} / d \theta=-74.2(7) \mathrm{kHz} / \mathrm{K}$ [32] for millimeter-sized samples. Low-temperature studies, down to $5 \mathrm{~K}$, show that this temperature dependence is, in fact, highly nonlinear; indeed, at liquid-nitrogen temperatures, linearizing yields a much weaker temperature dependence with $d D_{\mathrm{gs}} / d \theta \sim 100 \mathrm{~Hz} / \mathrm{K}[33]$. Finally, at higher temperatures (up to $600 \mathrm{~K}$ ), recent work has observed $\left|d D_{\mathrm{gs}} / d \theta\right|=100 \mathrm{kHz} / \mathrm{K}$, consistent with roomtemperature measurements [34]. The microscopic mechanism which underlies this pronounced zero-field splitting (ZFS) temperature dependence is not yet fully understood, but is thought to be related to the thermal expansion of the diamond. In particular, as temperatures vary, the local lattice spacing of the NV center is distorted causing changes in orbital overlaps which determine $D_{\mathrm{gs}}$. This picture is also consistent with very recent measurements of the room-temperature ZFS of single NV centers in bulk diamond, which also exhibits $d D_{\mathrm{gs}} / d \theta \sim-75 \mathrm{kHz} / \mathrm{K}$ [30]. Since the ensemble measurement is averaged over all NV orientations, the fact that it agrees identically with the single NV case suggests that crystallographic orientation does not affect $d D_{\mathrm{gs}} / d \theta$, consistent with our proposed mechanism which is owing to the overall thermal expansion of the lattice.

To further understand the effects of thermal expansion, we consider a schematic picture of the NV electronic orbitals; in particular, let us assume that the three orbitals associated with the basal carbon atoms are highly localized wave functions ( $\delta$ function), which simply move with their respective atoms. The microscopic origin of the zero-field splitting is thought to be spin-spin interactions, meaning that shifting of the wave functions will correspondingly alter the average magnetic dipole-dipole interaction, leading to a change in $D_{\mathrm{gs}}$. The dipole-dipole interaction is generally characterized as

$$
V_{i j} S_{i} S_{j}=-\frac{\kappa}{2}\left\langle\frac{3 \hat{r_{i}} \hat{r_{j}}-\delta_{i j}}{r^{3}}\right\rangle S_{i} S_{j},
$$

where $S_{i}$ is the magnetic dipole operator and $\kappa$ is the scale of the interaction strength $(2.88 \mathrm{GHz}$ at $2.6 \AA)$. To compute the above expectation value, we simply need to average over the displacements between each pair of carbon atoms. With 
$a$ as the distance between nearest-neighbor carbon atoms, the displacement vectors (without strain) are given by $\mathbf{r}^{(\mathbf{1})}=a \hat{y}$, $\mathbf{r}^{(2)}=\frac{\sqrt{3} a}{2} \hat{x}-\frac{a}{2} \hat{y}$, and $\mathbf{r}^{(3)}=-\frac{\sqrt{3} a}{2} \hat{x}-\frac{a}{2} \hat{y}$. Thus, the spin Hamiltonian in the absence of strain is simply

$$
H=V_{i j} S_{i} S_{j}=\frac{3 \kappa}{4 a^{3}}\left(S_{z}^{2}-2 / 3\right) .
$$

Under strain $\epsilon_{k l}$, the change in this Hamiltonian is

$$
\begin{aligned}
\Delta H= & S_{i} S_{j} \Delta V_{i j}=-\frac{\kappa}{2}\left\langle\left(\frac{\partial}{\partial r_{k}} \frac{3 \hat{r_{i}} \hat{r_{j}}-\delta_{i j}}{r^{3}}\right) \epsilon_{k l} \hat{r}_{l}\right\rangle S_{i} S_{j} \\
= & \frac{3 \kappa}{2 a^{3}}\left[-\frac{3}{4}\left(\epsilon_{x x}+\epsilon_{y y}\right)\left(S_{z}^{2}-2 / 3\right)\right. \\
& +\frac{1}{8}\left(\epsilon_{x x}-\epsilon_{y y}\right)\left(S_{x}^{2}-S_{y}^{2}\right)+\frac{1}{8}\left(\epsilon_{x y}+\epsilon_{y x}\right)\left(S_{x} S_{y}+S_{y} S_{x}\right) \\
& \left.-\frac{1}{2} \epsilon_{z x}\left(S_{x} S_{z}+S_{z} S_{x}\right)-\frac{1}{2} \epsilon_{z y}\left(S_{y} S_{z}+S_{z} S_{y}\right)\right] .
\end{aligned}
$$

Inspection reveals that the change in the ZFS is set by the coefficient of the first term, $\alpha=-\frac{3}{4}\left(\epsilon_{x x}+\epsilon_{y y}\right) \frac{3 \kappa}{2 a^{3}}$. In order to reproduce the zero strain ZFS of $\frac{3 \kappa}{4 a^{3}}=2.88 \mathrm{GHz}$, we take $a=2.38 \AA$, yielding $\alpha=-4.32 \mathrm{GHz}\left(\epsilon_{x x}+\epsilon_{y y}\right)$. Assuming isotropic expansion $\epsilon_{x x}=\epsilon_{y y}=\epsilon_{z z}$, our highly schematic model predicts a ZFS temperature dependence of

$$
\frac{d D_{\mathrm{gs}}}{d \theta}=(-4.32 \times 2)\left(\frac{1.6 \times 10^{-6}}{1 \mathrm{~K}}\right) \approx-15 \mathrm{kHz} / \mathrm{K},
$$

which is a factor of 5 from the observed room-temperature dependence [32]. This suggests that our proposed picture where temperature simply serves to distort the defect geometry is likely one of the dominant factors underlying the observed $\frac{d D_{\mathrm{gs}}}{d \theta}$. Such a temperature dependence under normal operating conditions makes this passive standard behave like a traditional crystal oscillator and poses a challenge for maintaining a precise operating temperature.

It is possible to stabilize the temperature of the diamond chip to within $0.01 \mathrm{~K}$ using commercially available Peltier coolers with proportional-integral-derivative (PID) control loops. This would, in principle, allow us to achieve $\approx 742 \mathrm{~Hz}$ uncertainty in the zero-field splitting, or a fractional frequency stability of $2.58 \times 10^{-7}$ at room temperature. We note that ensemble averaging would have no beneficial effect here, as all centers shift equally due to isotropic expansion. In the next section, building upon our discussions above, we will show that one might benefit by inducing anisotropy in the crystal's temperature response. Furthermore, if two different temperature dependencies can be identified, locking them in a feedback loop may also enable enhanced temperature stability.

\section{A. Temperature stabilization using synchronization}

Let us start by considering a scheme which utilizes two NV clocks. The zero-field splittings of the two clocks are assumed to have different temperature dependencies, a fact which can be achieved by utilizing two distinct diamond slabs mounted on substrates with different thermal expansion coefficients.

In particular, let us assume that one diamond slab ("1") is clamped in a stiff material (Young's modulus $E_{1}$ ) with a high thermal expansion coefficient $\eta_{c 1}$ (e.g., brass), while the second slab ("2") is clamped in a different material with Young's modulus $E_{2}$ and a lower thermal expansion coefficient $\eta_{c 2}<\eta_{c 1}$, as illustrated in Fig. 5(b). Both diamond slabs and clamps are assumed to be at the same temperature, $\theta$. Assuming that the clamps' cross-sectional area is much larger than that of the diamond slab, one finds that the change in strain imparted on the diamond can be approximated as $\Delta \epsilon_{1,2} \approx \eta_{d}\left(1+\eta_{c 1,2} E_{c 1,2} / E_{d}\right) \Delta \theta=\alpha_{1,2 \text { eff }} \Delta \theta$, where $\Delta \theta$ is the temperature difference from an initial set point $T_{0}$ and $\alpha_{\text {eff }}$ is the effective coefficient of thermal expansion. This set point should be chosen such that both clocks have the same initial frequency $\omega_{0}$ and can be adjusted by preloading strain within the samples, as illustrated in Fig. 5(a).

A conventional thermal feedback system maintains the temperature $\theta$ near $\theta_{0}$, but current thermistors only allow a long-term stability $\sim 10^{-2}-10^{-3} \mathrm{~K}$. To compensate for even smaller temperature drifts, we employ a protocol (see appendix for the detailed scheme) that exploits the different temperature dependencies of the two diamond samples. Such a "synchronized composite clock" has a modified frequency uncertainty:

$$
\frac{\Delta \omega_{1}^{\prime}}{\omega_{1}^{\prime}}=\left(\frac{\Delta \omega}{\omega}\right)_{\theta=\theta_{0}}\left[1+2\left(\frac{\beta_{1}}{\Delta \beta_{1,2}}\right)^{2}\right]^{1 / 2},
$$

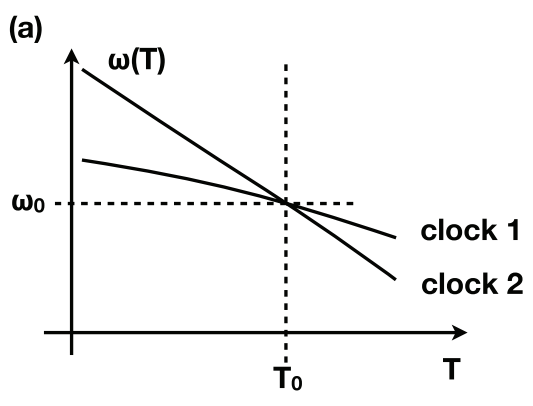

(b)

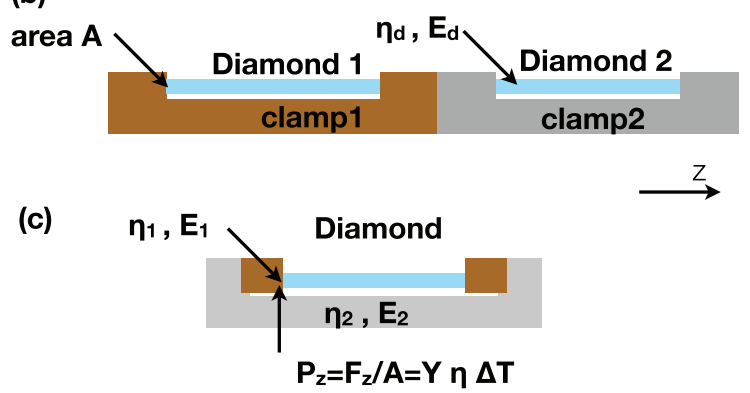

FIG. 5. (Color online) Temperature stabilization using two clocks. (a) The two diamond clocks have differing $d D_{\mathrm{gs}} / d \theta$ since they are clamped to substrates with different thermal expansion coefficients, $\eta_{1,2}$ and different Young's moduli, $E_{1,2}$. (b) Schematic illustration of a synchronized composite clock setup. (c) Alternatively, using strain engineering, a single clamp may be designed to fully cancel the temperature dependence of the NV ZFS. 
(a) $\Delta D_{\text {ZFS }}$ vs. Temperature

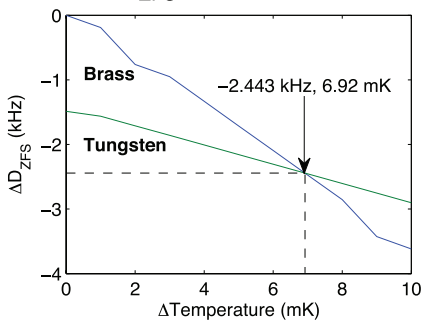

(b) $\quad \Delta D_{\text {ZFS }}$ vs. Position

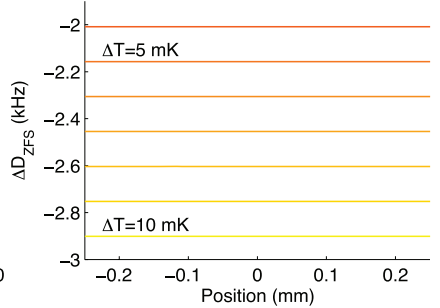

(c)

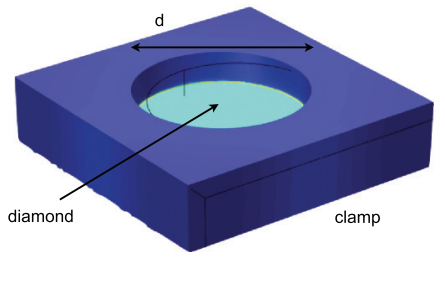

FIG. 6. (Color online) Simulated $D_{\text {gs }}$ variation with temperature for tungsten (green) and brass (blue) clamps. (b) Spatial frequency variation as a function of position within the diamond disk. (c) Clamp and diamond geometry.

where $\left(\frac{\Delta \omega}{\omega}\right)_{\theta=\theta_{0}}$ is the frequency uncertainty in the ideal limit of no microscopic temperature variations with the ensemble at $\theta_{0} . \beta_{1,2}=\alpha_{1,2 \text { eff }}\left(d D_{\mathrm{gs}} / d \epsilon\right)$ is the relative frequency shift per unit temperature and $\Delta \beta_{1,2}$ is the difference in these shifts.

The expression provides an intuitive result: The uncertainty is minimized when the temperature dependencies of the two clocks are different. In particular, Eq. (C5) reveals that when $\Delta \beta_{1,2} \gg \beta_{1}$, then the performance of the composite clock is similar to that of a bare temperature-insensitive NV clock. This stabilization is simplest if the two clocks are maintained at a frequency difference $v_{\text {beat }} \sim 10 \mathrm{kHz}$, so that the beat frequency can be locked to a high $Q\left(\sim 10^{6}\right)$ quartz oscillator.

Until now we have approximated that the strain imparted on the diamond by the clamp is uniform across the sample. To check the validity of this assumption, we model the composite system by finite element analysis as a circular disk of diamond clamped within a brass or tungsten holder, as shown in Fig. 6(c). Figure 6(a) depicts the calculated shift in $D_{\mathrm{gs}}$ as a function of temperature; the slopes agree with our previous expression for $\beta_{1,2} \sim \eta_{d}\left(1+\eta_{c 1,2} E_{c 1,2} / E_{d}\right) /(-75 \mathrm{kHz} / \mathrm{K})$. Finally, Fig. 6(b) shows that the strain is nearly uniform for a large area within the disk across the relevant temperature range $\Delta \theta \sim 0.01 \mathrm{~K}$, within the inner $1 \mathrm{~mm}$ diameter. Strain variation laterally (in-plane) is zero,within numerical precision, while vertically it is $10^{-12}$ because the diamond buckles. This strain corresponds to $\Delta D_{\mathrm{gs}}$ of $0.47 \mathrm{~Hz}$, or a fractional frequency deviation of $1.64 \times 10^{-10}$. Building upon the notion of altering the temperature dependence of the NV ZFS, we next consider the possibility of fully canceling the frequency dependence on temperature, $d D_{\mathrm{gs}} / d \theta$.

\section{B. Controlling temperature dependence with engineered strain}

As previously discussed, the temperature response of the NV $D_{\mathrm{gs}}$ likely originates from changes in the local strain field; in particular, fluctuations in temperature modulate the lattice, altering the position of the basal carbon atoms and thereby changing the effective spin-spin interaction. Within the usual ground state Hamiltonian, as expressed in Eq. (1), the strain dependence is hidden in the effective electric field vector $\vec{\sigma}$. In terms of actual components of the strain tensor $\epsilon$, perturbations to the spin Hamiltonian take the form $F_{i j k l} S_{i} S_{j} \epsilon_{k l}$, where $F$ is the fourth order strain response tensor. Symmetries reduce the number of allowed terms to eight totally symmetric combinations, and of these, we are interested in only the ones with an $S_{z}^{2}$ coefficient. Thus, one finds that the strain dependence of the ZFS is most generally characterized as

$$
\left[D_{\mathrm{gs}}+A_{1}\left(\epsilon_{x x}+\epsilon_{y y}\right)+A_{2} \epsilon_{z z}\right]\left(S_{z}^{2}-2 / 3\right),
$$

where $A_{1}$ and $A_{2}$ are parameters which require further experimental input. In the case of isotropic expansion $\epsilon_{x x}=\epsilon_{y y}=$ $\epsilon_{z z}$, one would expect $d D_{\mathrm{gs}} / d \theta=2 A_{1}+A_{2}$. By clamping the diamond along a specific direction, one can envision inducing an anisotropic lattice response which may significantly reduce the effective temperature dependence of the ZFS.

A schematic example is illustrated in Fig. 6(b). Consider a small temperature shift $\Delta \theta$, near $\theta_{0}$, which would cause a diamond slab of length $L_{d}$ to expand by $\Delta L=L_{d} \eta_{d} \Delta \theta$. If the diamond slab were instead clamped, this expansion could easily be modulated. For instance, if material 2, which forms the bottom of the clamp, has a low thermal expansion coefficient and high Young's modulus, and material 1 has a high thermal expansion coefficient and high Young's modulus, then a pressure $P=E_{d} \Delta L_{d} / L=E_{d} \varepsilon_{d}=E_{d} \eta_{d} \Delta \theta$ may be exerted to compress the diamond slab by $-\Delta L$. For temperature changes $\Delta \theta \sim 0.01 \mathrm{~K}$, this pressure is $\sim 12.2 \mathrm{kPa}$. One possible material combination (among many), is to use brass for material 1 and silicon carbide for material 2; tuning of the modulated temperature can then be achieved by altering the width of the brass layer.

\section{CONCLUSIONS}

In summary, we have proposed a solid-state spin-optical frequency standard based on the NV defect center in diamond. Our analysis reveals that the diamond frequency standard can potentially yield a fully chip-integrable solid-state timekeeping platform whose performance rivals that of modern atomic clocks, both laboratory and chip scale. A proposed device is shown in Fig. 4 and contains a diamond chip grown with a dielectric cavity for lowering the optical pumping power requirements, PL collection with on-chip Si photodiodes, and planar microwave waveguides for addressing the NV transitions, all of which can be integrated within modern device fabrication. Such miniaturized clocks can easily be integrated into scientific and consumer electronics and could be used in harsh external environments. Devices benefiting include those for wireless communication and GPS navigation (with improved tolerance to jamming) [36]. In addition, the clock's center frequency of $2.870 \mathrm{GHz}$ could enable a wide-bandwidth data rate in next-generation cellular communications $(f \geqslant 40 \mathrm{GHz})$. 
Due to the center's relatively long lifetime, high density of spins, and optical detection, we estimate a time stability exceeding $\sigma_{y}=2 \times 10^{-12} \tau^{-1 / 2}$, rivaling that of the newest chip-scale $\mathrm{Cs}$ and $\mathrm{Rb}$ standards over short times: seconds to minutes. Many of the technological advances, such as surface emitting lasers and chip-scale detectors, are readily deployable to this system, with the added benefit that the atomic system resides within the diamond substrate. We anticipate the ability to greatly improve upon this frequency standard using repetitive readout [21], entanglement [37], IR absorption detection [38], and nanophotonics [39].

\section{ACKNOWLEDGMENTS}

The authors acknowledge Peter Maurer, Colm Ryan, Peter Kinget, and Tanya Zelevinsky for useful discussions and Hannah Clevenson for comments on the manuscript. This work was supported by the NSF, CUA, Packard Foundation, DOE (FG02-97ER25308), the Air Force Office of Scientific Research PECASE award (FA9550-12-0045), and the 2012 DARPA YFA program (N66001-13-1-4027).

\section{APPENDIX A: STEADY-STATE RESPONSE OF NV- CENTER UNDER EXCITATION}

The spin and orbital energy levels of the negatively charged nitrogen-vacancy center $\left(\mathrm{NV}^{-}\right)$has been well understood for decades, but continues to attract interest and revision due to a wealth of precision experiments which continue to elucidate the spin and orbital contributions of the triplet and singlet levels at both room and cryogenic temperatures. For the purpose of a room-temperature frequency standard, we adopt the well-accepted "seven-level model" of the system [16] and the rates put forth in this work [40]. We label the states according to the energy diagram in Fig. 1(a). Note that the eigenstates $|-1\rangle$ and $|+1\rangle$ are degenerate in this picture and comprise both the levels $\mathbf{1}$ and $\mathbf{3}$ in the ground and excited states, respectively. Without loss of generality, we omitted the $|-1\rangle$ level, deviating from the full Hamiltonian in the main text. This can be rationalized in one of two ways: (i) the microwave excitations are circularly polarized, thus only the transitions $|+1\rangle$ are allowed by selection rules [41]; or (ii) a small bias magnetic field is applied to split the $| \pm 1\rangle$ degeneracy and under a rotating wave approximation with limited microwave excitation power the $|-1\rangle$ levels are far from resonance. In either of these cases the rotating frame Hamiltonian in the subspace is

$$
h_{\mathrm{gs}}=\Delta(|1\rangle\langle 1|-| 0\rangle\langle 0|)+\Omega(|1\rangle\langle 0|+| 0\rangle\langle 1|),
$$

where $\boldsymbol{\Omega}$ is the microwave Rabi frequency and $\boldsymbol{\Delta}$ is the microwave detuning from resonance. The other rates defined in the energy-level diagram are $\mathbf{I}$, the optical pumping rate; $\gamma$, the spontaneous emission rate of the excited state triplet (assumed to be wavelength independent); $\kappa$, the intersystem crossing rate; $\lambda$, the deshelving rate from the singlet to the $\left|m_{s}=0\right\rangle$ ground state; $\epsilon$, the fractional spin mixing of the optical excitation; $\boldsymbol{\Gamma}_{\mathbf{1}}$, the rate of spin flip from 1 to $0 ; \boldsymbol{\Gamma}_{\mathbf{2}}$, the rate of spin flip from 0 to 1 ; and $\boldsymbol{\Gamma}_{\mathbf{3}}$, the dephasing of the spin. Relaxation from $|2\rangle$ to $|s\rangle$ is considered nominally forbidden and can be incorporated in the $\epsilon$ constant.
We incorporate these rates into a Lindblad master equation:

$$
\dot{\rho}=\frac{1}{i \hbar}\left[H_{\mathrm{gs}}, \rho\right]+\sum_{k} L_{k} \rho L_{k}^{\dagger}-\frac{1}{2} L_{k}^{\dagger} L_{k} \rho-\frac{1}{2} \rho L_{k}^{\dagger} L_{k}
$$

for the density matrix of the system, $\rho$.

We assume the following set of jump operators for the optical pumping and relaxation processes:

$$
\begin{aligned}
L_{1} & =\sqrt{I}|2\rangle\left\langle 0\left|, \quad L_{2}=\sqrt{I}\right| 3\right\rangle\left\langle 1\left|, \quad L_{3}=\sqrt{\epsilon}\right| 3\right\rangle\langle 0|, \\
L_{4} & =\sqrt{\kappa}|s\rangle\left\langle 3\left|, \quad L_{5}=\sqrt{\lambda}\right| 0\right\rangle\left\langle s\left|, \quad L_{6}=\sqrt{\gamma}\right| 0\right\rangle\langle 2|, \\
L_{7} & =\sqrt{\gamma}|2\rangle\left\langle 3\left|, \quad L_{8}=\sqrt{\Gamma_{1}}\right| 0\right\rangle\left\langle 1\left|, \quad L_{9}=\sqrt{\Gamma_{2}}\right| 1\right\rangle\langle 0|, \\
L_{10} & =\sqrt{\Gamma_{3}}(|1\rangle\langle 1|-| 0\rangle\langle 0|) .
\end{aligned}
$$

Adding in the Hamiltonian and gathering the terms for each matrix element of $\rho$, we arrive at the following system of differential equations:

$$
\begin{aligned}
\dot{\rho}_{00}= & -i \Omega\left(\rho_{10}-\rho_{01}\right)-I(1+\epsilon) \rho_{00}+\lambda \rho_{\mathrm{ss}} \\
& +\gamma \rho_{22}+\Gamma_{1} \rho_{11}-\Gamma_{2} \rho_{00},
\end{aligned}
$$

$$
\dot{\rho}_{11}=-i \Omega\left(\rho_{01}-\rho_{10}\right)-I \rho_{11}+\gamma \rho_{33}-\Gamma_{1} \rho_{11}+\Gamma_{2} \rho_{00},
$$

$$
\begin{gathered}
\dot{\rho}_{01}=-2 i \Delta \rho_{01}+i \Omega\left(\rho_{00}-\rho_{11}\right)-I(1+\epsilon / 2) \rho_{01} \\
-\left(\frac{\Gamma_{1}}{2}+\frac{\Gamma_{2}}{2}+2 \Gamma_{3}\right) \rho_{01}, \\
\dot{\rho}_{10}=2 i \Delta \rho_{10}+i \Omega\left(\rho_{11}-\rho_{00}\right)-I(1+\epsilon / 2) \rho_{10} \\
-\left(\frac{\Gamma_{1}}{2}+\frac{\Gamma_{2}}{2}+2 \Gamma_{3}\right) \rho_{10}, \\
\dot{\rho}_{22}=I \rho_{00}-\gamma \rho_{22}, \\
\dot{\rho}_{33}=I \rho_{11}+I \epsilon \rho_{00}-(\gamma+\kappa) \rho_{33}, \\
\dot{\rho}_{\mathrm{ss}}=-\lambda \rho_{\mathrm{ss}}+\kappa \rho_{33},
\end{gathered}
$$

where $\rho_{j k}=|j\rangle\langle k|$ and $\hbar=1$.

Note that this is not a complete set of differential equations for all 25 matrix elements of our five-level system. We make the key assumption that coherences (off-diagonal matrix elements) only exist in the ground state and that all other matrix elements can be neglected as they decay away much faster than the other dynamics of the system. We also need the system to satisfy the unit probability of the density matrix (i.e., $\operatorname{Tr}[\rho]=1$ ), which gives us the final equation to completely solve the system:

$$
\rho_{00}+\rho_{11}+\rho_{22}+\rho_{33}+\rho_{\mathrm{ss}}=1 .
$$

When seeking to use the magnetic transition of the electron spin sublevels as our clock levels, we primarily care about the steady-state dynamics of the system for irradiation times much longer than the longest relaxation time in the system. In this case, we take our equations above and set $\dot{\rho}_{j k} \rightarrow 0$.

To arrive at a meaningful answer, we start by defining the variables:

$$
a=I(1+\epsilon / 2)+\frac{\Gamma_{1}+\Gamma_{2}}{2}+2 \Gamma_{3}, \quad b=2 i \Delta, \quad c=i \Omega .
$$


After a bit of algebra, we arrive at the steady-state solutions for the populations responsible for the signal:

$$
\begin{gathered}
\left(\frac{\gamma}{I}(1-\epsilon)+1\right) \rho_{22}^{\mathrm{ss}}=1-\left(\frac{\kappa}{\gamma}+\frac{\gamma+\kappa}{I}+1\right) \rho_{33}^{\mathrm{ss}}, \\
\left(\epsilon \gamma+\frac{\gamma\left(\Gamma_{2}+\epsilon \Gamma_{1}\right)}{I}-(1+\epsilon) \frac{\gamma}{I} \frac{2 a c^{2}}{a^{2}-b^{2}}\right) \rho_{22}^{\mathrm{ss}} \\
=\left(\kappa+\frac{\Gamma_{1}(\kappa+\gamma)}{I}-\frac{\gamma}{I} \frac{2 a c^{2}}{a^{2}-b^{2}}\right) \rho_{33}^{\mathrm{ss}} .
\end{gathered}
$$

Making another substitution for these variables, we define

$$
\begin{aligned}
& p=\left(\frac{\gamma}{I}(1-\epsilon)+1\right), \\
& q=\left(\frac{\kappa}{\gamma}+\frac{\gamma+\kappa}{I}+1\right), \\
& f=\left(\epsilon \gamma+\frac{\gamma\left(\Gamma_{2}+\epsilon \Gamma_{1}\right)}{I}-(1+\epsilon) \frac{\gamma}{I} \frac{2 a c^{2}}{a^{2}-b^{2}}\right), \\
& g=\left(\kappa+\frac{\Gamma_{1}(\kappa+\gamma)}{I}-\frac{\gamma}{I} \frac{2 a c^{2}}{a^{2}-b^{2}}\right),
\end{aligned}
$$

and arrive at the steady-state populations $\rho_{22}^{\text {ss }}$ and $\rho_{33}^{\text {ss }}$ in terms of $f, g, p$, and $q$ :

$$
\begin{aligned}
& \rho_{33}^{\mathrm{ss}}=\frac{f}{f q+p g}, \\
& \rho_{22}^{\mathrm{ss}}=\frac{g}{f q+p g} .
\end{aligned}
$$

The observed signal is proportional to the excited state populations weighted by the branching ratio and normalized to the emission rate:

$$
F(\Delta)=\gamma\left(\rho_{22}^{\mathrm{ss}}(\Delta)+\frac{\gamma}{\kappa+\gamma} \rho_{33}^{\mathrm{ss}}(\Delta)\right) .
$$

This can be cast into a single Lorentzian function of the form

$$
F(\Delta)=I_{0}\left(1-\frac{C \delta v^{2}}{(\Delta / \pi)^{2}+\delta v^{2}}\right),
$$

where $C$ is the modulation depth and $\delta v$ is the FWHM. Values quoted in the main text were obtained by numerical calculation of the steady-state master equation for a variety of detuning and fitting for the parameters $C$ and $\delta v$.

\section{APPENDIX B: MEASUREMENT AND SENSITIVITY}

The accuracy of any frequency standard in the pulsed Ramsey or echo prescription is described by the deviation in the fractional frequency, $\frac{\delta v}{\nu_{0}}$. At the point of maximum slope of the variation of the observed signal, $\langle\hat{M}\rangle$, with respect to the acquired phase $\varphi=\delta \Omega \tau=2 \pi \tau\left(v_{0}-v\right)$, this fractional frequency is

$$
\frac{\delta v}{v_{0}}=\frac{1}{v_{0}} \frac{\Delta \hat{M}}{|\partial\langle\hat{M}\rangle / \partial \nu|} .
$$

In order to evaluate this expression, we must know the observable signal-to-noise ratio for the NV measurement and follow the measurement operator described in [20] for the
NV spin-dependent fluorescence readout. Let us take the measurement operator to be

$$
\hat{M}=a|0\rangle\langle 0|+b(|+1\rangle\langle+1|+|-1\rangle\langle-1|),
$$

where $a$ and $b$ are random Poisson variables. This prescription of a randomized measurement operator follows from the observation that if the measured state is $|\psi\rangle=|0\rangle$, the average number of photons detected in a measurement period is $\langle a\rangle=\alpha$, with variance roughly equal to $\alpha$ after $\sim 10^{5}$ repeated measurements. Likewise, if the measured state is $|\psi\rangle=| \pm 1\rangle$, the average number of photons detected in a measurement period is $\langle b\rangle=\beta$. Due to poor collection efficiency, $\alpha$ and $\beta \ll 1$. Note that although $\hat{M}$ is diagonal it is not idempotent, the consequences of which we shall see below.

Given the expression in Eq. (6), we can then calculate the moments of $\hat{M}$.

$$
\begin{gathered}
\langle\hat{M}\rangle=\alpha \sin ^{2}(\varphi)+\beta \cos ^{2}(\varphi), \\
\left\langle\hat{M}^{2}\right\rangle=\alpha(\alpha+1) \sin ^{2}(\varphi)+\beta(\beta+1) \cos ^{2}(\varphi) .
\end{gathered}
$$

From algebraic combinations, we can get the variance of $\hat{M}$ :

$$
\begin{aligned}
\Delta \hat{M}^{2}= & \left\langle\hat{M}^{2}\right\rangle-\langle\hat{M}\rangle^{2} \\
= & \left(\frac{\alpha+\beta}{2}\right)^{2} \sin ^{2}(2 \varphi)-\left(\frac{\alpha-\beta}{2}\right) \cos ^{2}(2 \varphi) \\
& +\left(\frac{\alpha+\beta}{2}\right)
\end{aligned}
$$

and the derivative of $\langle\hat{M}\rangle$ :

$$
\left|\frac{\partial\langle\hat{M}\rangle}{\partial v}\right|=2 \pi \tau(\alpha-\beta) \sin (2 \varphi) .
$$

The NV center at room temperature has a count rate with only a marginal discrimination between the $|0\rangle$ and $| \pm 1\rangle$ states, with $2 \alpha \approx 3 \beta$, due to the imperfect spin measurement. This sets an upper bound on the fractional frequency:

$$
\left(\frac{\delta v}{v_{0}}\right)_{\mathrm{NV}}=\frac{\xi}{2 \pi v_{0} \tau}
$$

with

$$
\xi=\frac{\sqrt{\frac{25}{36} \alpha^{2} \sin ^{2}(2 \varphi)+\frac{5}{3} \alpha-\frac{1}{3} \alpha \cos (2 \varphi)}}{\frac{1}{3} \alpha \sin (2 \varphi)} .
$$

For the $\pi / 4$ point, $\xi_{\pi / 4}=\frac{25}{12} \alpha+5 \approx 5$, as $\alpha=0.03$. Note that for single ion microwave clocks, $\xi \approx 1$. Thus, the imperfect spin measurement, comprised of optical pumping of the spin state and nonzero fluorescence from all eigenstates, adds a factor of 5 over the idealized measurements for long-shelved ion states.

If we have an ensemble of $N \mathrm{NV}$ centers and make $M$ measurements in a total interrogation time $T=M \tau$, the fractional frequency reduces by a factor of $\sqrt{M N}$ :

$$
\left\langle\frac{\delta v}{v_{0}}\right\rangle_{M, N}=\frac{\xi}{2 \pi v_{0} \sqrt{\tau N T}} .
$$


Phase accumulation under the echo sequence can extend to our $\tau=T_{2} \approx 1 \mathrm{~ms}$. With $v_{0}=2870 \mathrm{MHz}$ this gives the sensitivity of $\delta v / \nu_{0}=8.8 \times 10^{-9} / \sqrt{N T}$.

\section{APPENDIX C: TWO-CLOCK STABILIZATION SCHEME}

Our temperature stabilization scheme seeks to exploit the difference in temperature dependencies imposed on two diamond samples whose resonance frequencies $\omega_{1}$ and $\omega_{2}$, vary differently with temperature due to unequal boundary conditions imposed by dissimilar materials. We define the two resonance frequencies as

$$
\begin{aligned}
& \omega_{1}(\theta)=\omega_{0}+\beta_{1} \Delta \theta, \\
& \omega_{2}(\theta)=\omega_{0}+\beta_{2} \Delta \theta,
\end{aligned}
$$

where $\beta_{1,2}=\frac{d \epsilon}{d \theta} \frac{d D_{\mathrm{gs}}}{d \epsilon}=\eta_{d}\left(1+\eta_{c 1,2} E_{c 1,2} / E_{d}\right)\left(d D_{\mathrm{gs}} / d \epsilon\right)$. At time $\tau=0$, both clocks are at the same temperature $\theta_{0}$, before the temperature is allowed to fluctuate within a small range around $\theta_{0}$. After time $t$, these clocks have acquired a phase

$$
\phi_{1,2}(t)=\omega_{0} t+\int_{0}^{t} \beta_{1,2} \Delta \theta\left(t^{\prime}\right) d t^{\prime} \pm \Delta \phi_{0},
$$

where $\Delta \phi_{0}=\xi \sqrt{t} / \sqrt{\theta_{2, \text { ens }} N}$ is the phase uncertainty. The difference between the two phases may be recorded by mixing and low-pass filtering the two clock signals, giving $\Delta \phi(t)=$ $\phi_{2}(t)-\phi_{1}(t)=\int_{0}^{t} \Delta \beta_{1,2} \Delta \theta\left(t^{\prime}\right) d t^{\prime} \pm \sqrt{2} \Delta \phi_{0}$, where $\Delta \beta_{1,2} \equiv$ $\beta_{2}-\beta_{1}$ and we have assumed identical variances for $\phi_{1}(t)$ and $\phi_{2}(t)$.

Now, one can "correct" for temperature fluctuations in clock 1:

$$
\begin{aligned}
\phi_{1}^{\prime}(t) & =\phi_{1}(t)-\int_{0}^{t} \beta_{1} \Delta \theta\left(t^{\prime}\right) d t^{\prime} \\
& =\phi_{1}(t)-\left(\int_{0}^{t} \Delta \beta_{1,2} \Delta \theta\left(t^{\prime}\right) d t^{\prime}\right) \frac{\beta_{1}}{\Delta \beta_{1,2}} \\
& =\phi_{1}(t)-\frac{\beta_{1}}{\Delta \beta_{1,2}}\left(\Delta \phi(t) \mp \sqrt{2} \Delta \phi_{0}\right) .
\end{aligned}
$$

Dividing by $t$ yields the frequency, from which we then find the uncertainty of the new "synchronized composite clock" of $N \mathrm{NV}$ centers after a time $t$ :

$$
\begin{aligned}
\frac{\Delta \omega_{1}^{\prime}}{\omega_{1}^{\prime}} & =\frac{\xi}{\sqrt{T_{2} N t} D_{\mathrm{gs}}}\left[1+2\left(\frac{\beta_{1}}{\Delta \beta_{1,2}}\right)^{2}\right]^{1 / 2} \\
& =\left(\frac{\Delta \omega}{\omega}\right)_{\theta=\theta_{0}}\left[1+2\left(\frac{\beta_{1}}{\Delta \beta_{1,2}}\right)^{2}\right]^{1 / 2} .
\end{aligned}
$$

[1] J. Camparo, Phys. Today 60(11), 33 (2007).

[2] T. Rosenband, D. B. Hume, P. O. Schmidt, C. W. Chou, A. Brusch, L. Lorini, W. H. Oskay, R. E. Drullinger, T. M. Fortier, J. E. Stalnaker et al., Science 319, 1808 (2008).

[3] M. Takamoto, F.-L. Hong, R. Higashi, and H. Katori, Nature (London) 435, 321 (2005).

[4] A. D. Ludlow, T. Zelevinsky, G. K. Campbell, S. Blatt, M. M. Boyd, M. H. G. de Miranda, M. J. Martin, J. W. Thomsen, S. M. Foreman, J. Ye et al., Science 319, 1805 (2008).

[5] C. W. Chou, D. B. Hume, T. Rosenband, and D. J. Wineland, Science 329, 1630 (2010).

[6] F. Riehle, Frequency Standards: Basics and Applications (Wiley-VCH, Weinheim, 2004).

[7] S. Knappe, V. Shah, P. D. D. Schwindt, L. Hollberg, J. Kitching, L.-A. Liew, and J. Moreland, Appl. Phys. Lett. 85, 1460 (2004).

[8] D. Youngner, L. Lust, D. Carlson, S. Lu, L. Forner, H. Chanhvongsak, and T. Stark, in Proceedings of the IEEE Conference on Solid-State Sensors, Actuators and Microsystems, 2007 (unpublished), pp. 39-44.

[9] V. Shah, S. Knappe, P. D. D. Schwindt, and J. Kitching, Nat. Photonics 1, 649 (2007).

[10] D. M. Toyli, C. D. Weis, G. D. Fuchs, T. Schenkel, and D. D. Awschalom, Nano Lett. 10, 3168 (2010).

[11] G. Balasubramanian, P. Neumann, D. Twitchen, M. Markham, R. Kolesov, N. Mizuochi, J. Isoya, J. Achard, J. Beck, J. Tissler et al., Nature Mater. 8, 383 (2009).

[12] C. White and A. Hajimiri, in Proceedings of the IEEE Frequency Control Symposium and Exposition, 2005 (unpublished).

[13] L. Childress, M. V. Gurudev Dutt, J. M. Taylor, A. S. Zibrov, F. Jelezko, J. Wrachtrup, P. R. Hemmer, and M. D. Lukin, Science 314, 281 (2006).
[14] F. Dolde, H. Fedder, M. W. Doherty, T. Nobauer, F. Rempp, G. Balasubramanian, T. Wolf, F. Reinhard, L. C. L. Hollenberg, F. Jelezko et al., Nat. Phys. 7, 459 (2011).

[15] Note that the $g$ factor for the $\mathrm{NV}^{-}$center has been shown, via ensemble measurements, to be slightly anisotropic [42], with $g_{\|}=2.0029(2)$ and $g_{\perp}=2.0031(2)$. This does not affect the clock protocol for two reasons: (i) the magnetic field used to split the resonance is along the direction of the NV symmetry axis whereby only the $z$ term would be relevant; and (ii) in the pulsed protocol, the Zeeman terms of the Hamiltonian are averaged away to lowest order.

[16] N. B. Manson, J. P. Harrison, and M. J. Sellars, Phys. Rev. B 74, 104303 (2006).

[17] J. R. Maze, P. L. Stanwix, J. S. Hodges, S. Hong, J. M. Taylor, P. Cappellaro, L. Jiang, M. V. G. Dutt, E. Togan, A. S. Zibrov et al., Nature (London) 455, 644 (2008).

[18] A. Dréau, M. Lesik, L. Rondin, P. Spinicelli, O. Arcizet, J.-F. Roch, and V. Jacques, Phys. Rev. B 84, 195204 (2011).

[19] I. Chan, A. Andreyuk, S. Beattie, B. Barrett, C. Mok, M. Weel, and A. Kumarakrishnan, Phys. Rev. A 78, 033418 (2008).

[20] C. A. Meriles, L. Jiang, G. Goldstein, J. S. Hodges, J. Maze, M. D. Lukin, and P. Cappellaro, J. Chem. Phys. 133, 124105 (2010).

[21] L. Jiang, J. S. Hodges, J. R. Maze, P. Maurer, J. M. Taylor, D. G. Cory, P. R. Hemmer, R. L. Walsworth, A. Yacoby, A. S. Zibrov et al., Science 326, 267 (2009).

[22] D. Le Sage, L. M. Pham, N. Bar-Gill, C. Belthangady, M. D. Lukin, A. Yacoby, and R. L. Walsworth, Phys. Rev. B 85, 121202 (2012). 
[23] G. de Lange, Z. H. Wang, D. Ristè, V. V. Dobrovitski, and R. Hanson, Science 330, 60 (2010).

[24] C. A. Ryan, J. S. Hodges, and D. G. Cory, Phys. Rev. Lett. 105, 200402 (2010).

[25] P. C. Maurer, G. Kucsko, C. Latta, L. Jiang, N. Y. Yao, S. D. Bennett, F. Pastawski, D. Hunger, N. Chisholm, M. Markham et al., Science 336, 1283 (2012).

[26] J. M. Taylor, P. Cappellaro, L. Childress, L. Jiang, D. Budker, P. R. Hemmer, A. Yacoby, R. Walsworth, and M. D. Lukin, Nat. Phys. 4, 810 (2008).

[27] P. Spinicelli, A. Dréau, L. Rondin, F. Silva, J. Achard, S. Xavier, S. Bansropun, T. Debuisschert, S. Pezzagna, J. Meijer et al., New J. Phys. 13, 025014 (2011).

[28] C. P. Slichter, Principles of Magnetic Resonance, 3rd ed. (Springer-Verlag, Berlin, 1996).

[29] P. Stanwix, L. Pham, J. Maze, D. L. Sage, T. Yeung, P. Cappellaro, P. Hemmer, A. Yacoby, M. Lukin, and R. Walsworth, Phys. Rev. B 82, 201201 (2010).

[30] P. C. Maurer (unpublished).

[31] P. M. Martineau, M. P. Gaukroger, K. B. Guy, S. C. Lawson, D. J. Twitchen, I. Friel, J. O. Hansen, G. C. Summerton, T. P. G. Addison, and R. Burns, J. Phys.: Condens. Matter 21, 364205 (2009).

[32] V. M. Acosta, E. Bauch, M. P. Ledbetter, A. Waxman, L.-S. Bouchard, and D. Budker, Phys. Rev. Lett. 104, 070801 (2010).
[33] X.-D. Chen, C.-H. Dong, F.-W. Sun, C.-L. Zou, J.-M. Cui, Z.-F. Han, and G.-C. Guo, Appl. Phys. Lett. 99, 161903 (2011).

[34] D. M. Toyli, D. J. Christle, A. Alkauskas, B. Buckley, C. G. Van de Walle, and D. D. Awschalom, Phys. Rev. X 2, 031001 (2012).

[35] G. A. Kazakov, A. N. Litvinov, V. I. Romanenko, L. P. Yatsenko, A. V. Romanenko, M. Schreitl, G. Winkler, and T. Schumm, New J. Phys. 14, 083019 (2012).

[36] J. Vig, IEEE Trans. Ultrason. Ferroelectr. Freq. Control 40, 522 (1993).

[37] P. Neumann, J. Beck, M. Steiner, F. Rempp, H. Fedder, P. R. Hemmer, J. Wrachtrup, and F. Jelezko, Science 329, 542 (2010).

[38] V. M. Acosta, A. Jarmola, E. Bauch, and D. Budker, Phys. Rev. B 82, 201202 (2010).

[39] P. E. Barclay, K.-M. C. Fu, C. Santori, and R. G. Beausoleil, Appl. Phys. Lett. 95, 191115 (2009).

[40] Recent experiments [18,43] have called some of these rates into question. These do not greatly impact the heuristic operation of the clock, nor its precision.

[41] Thiago P. Mayer Alegre, C. Santori, G. Medeiros-Ribeiro, and R. G. Beausoleil, Phys. Rev. B 76, 165205 (2007).

[42] S. Felton, A. M. Edmonds, M. E. Newton, P. M. Martineau, D. Fisher, D. J. Twitchen, and J. M. Baker, Phys. Rev. B 79, 075203 (2009).

[43] L. Robledo, H. Bernien, T. van der Sar, and R. Hanson, New J. Phys. 13, 025013 (2011). 